\title{
Aortic wall inflammation: a more accurate predictor of aneurysm expansion and aneurysm rupture risk than aortic diameter?
}

\author{
Kosmas I. Paraskevas \\ Department of Vascular Surgery, Royal Free Hospital, Royal Free London NHS Foundation Trust, London, UK \\ Correspondence to: Kosmas I. Paraskevas, MD, PhD. Department of Vascular Surgery, Royal Free Hospital, Pond Street, London NW3 2QG, UK. \\ Email: paraskevask@hotmail.com. \\ Provenance: This is an invited Editorial commissioned by the Section Editor Lei Zhang (Department of Vascular Surgery, Changhai Hospital, Second \\ Military Medical University, Shanghai, China). \\ Comment on: MA3RS Study Investigators. Aortic Wall Inflammation Predicts Abdominal Aortic Aneurysm Expansion, Rupture, and Need for \\ Surgical Repair. Circulation 2017;136:787-97.
}

Submitted Jul 24, 2018. Accepted for publication Sep 11, 2018.

doi: 10.21037/jtd.2018.09.36

View this article at: http://dx.doi.org/10.21037/jtd.2018.09.36

According to the 2018 Society for Vascular Surgery recommendations regarding the management of patients with abdominal aortic aneurysms (AAAs), elective repair is strongly recommended for the patients at an average risk for surgery with a fusiform $\mathrm{AAA} \geq 5.5 \mathrm{~cm}$ in diameter [level of recommendation: 1 (strong); quality of evidence: A (high)] (1). A weaker recommendation is provided for patients with saccular aneurysms of any size [level of recommendation: 2 (weak); quality of evidence: C (low)], as well as for females with an AAA that is $5.0-5.4 \mathrm{~cm}$ in maximum diameter [level of recommendation: 2 (weak); quality of evidence: B (moderate)] (1).

Traditionally, the maximum AAA diameter has been considered as the main criterion to consider an elective repair. The 5.5 -cm-diameter threshold was not selected randomly. It has been demonstrated that when the diameter of AAAs exceeds the $5.5 \mathrm{~cm}$ threshold, their risk of rupture is greater than the perioperative mortality which is associated with an open surgical AAA repair. Nevertheless, a maximum AAA diameter of $5.5 \mathrm{~cm}$ is not an absolute criterion indicating AAA rupture risk. Some AAAs (particularly in females) rupture at a smaller diameter, whereas others expand to $7.0,8.0$ or even $>9.0 \mathrm{~cm}$ without rupturing. In a 20 -year retrospective analysis of a singlecentre AAA surveillance database ( $\mathrm{n}=692$ patients), 3.9\% of patients in surveillance ruptured and $40.7 \%$ of those had a maximum anteroposterior diameter below $5.5 \mathrm{~cm}$ in diameter (2). On the other side, a study assessing the survival rates of AAA in patients who were deemed unfit for an elective repair showed that the mean AAA diameter in those patients that died of AAA rupture was 7.4 (range, $6.2-8.5) \mathrm{cm}$ (3). This suggests that other prognostic factors need to be identified able to predict more accurately AAA expansion and AAA rupture risk.

The recently published, multicenter $M^{3}{ }^{3}$ RS study [magnetic resonance imaging (MRI) using ultrasound superparamagnetic particles of iron oxide to predict clinical outcome in patients under surveillance for AAAs] prospectively enrolled patients who were under regular ultrasound surveillance for their AAA (4). The purpose of this trial was the measurement of ultrasmall superparamagnetic particles of iron oxide (USPIO), which is a class of MRI contrast agent to be taken up by tissueresident macrophages. USPIO may identify inflammation at the cellular level within tissues including AAA (4). Patients $(n=342)$ were recruited from 3 centers in Scotland, who had a maximum anteroposterior AAA diameter of $\geq 40 \mathrm{~mm}$ by abdominal ultrasound. Besides a full clinical assessment, each patient received a USPIO-enhanced MRI and a computed tomography aortography.

A total of 146 participants $(42.7 \%$ of the cohort) had demonstrated a USPIO enhancement of the AAA, whereas another 191 patients (55.8\% of the group) had no USPIO enhancement and in 5 patients $(1.5 \%$ of the study participants) it was indeterminate (4). The authors found a strong association between the USPIO enhancement and 
the current smoking status as well as with baseline AAA size and the presence of a common iliac aneurysm. The average AAA growth rate during the study was $2.8 \pm 2.4 \mathrm{~mm} /$ year $(\mathrm{n}=279)$ and was found to be greater in patients with USPIO enhancement $[3.1 \pm 2.5$ vs. $2.5 \pm 2.4 \mathrm{~mm} /$ year; difference: $0.6 \mathrm{~mm} /$ year; $95 \%$ confidence interval (CI): 0.02-1.2; $\mathrm{P}=0.0424]$. The composite of an AAA rupture or AAA repair occurred more frequently in participants with USPIO enhancement of AAA (69 of 146 vs. 68 of 191 patients, or $47.3 \%$ vs. $35.6 \%$, respectively; difference: $11.7 \%$, 95\% CI: $1.1-22.2 \% ; \mathrm{P}=0.0308)$. Patients with USPIO enhancements also had a reduced event-free survival $(\mathrm{P}=0.0275)$. Patients with smaller AAAs had lower rates of USPIO enhancements when compared with patients with larger AAAs [65 (35.1\%) vs. 81 (53.3\%); difference: $18.2 \%$; 95\% CI: 7.7-28.9\%; $\mathrm{P}=0.0008$ ] (4). Patients with smaller aneurysms demonstrating USPIO enhancement had double the rates of AAA repair or rupture. In contrast, those individuals with larger AAAs had demonstrated USPIO enhancement, the reverse occurred, with a more than doubling of mortality but no effect on the primary end point. The conclusion reached was that USPIO-enhanced MRI predicts the rate of AAA expansion, as well as the risk of AAA rupture and AAA repair (4).

The theory that inflammation may predict AAA rupture risk is not novel. Inflammation plays a key role in the development of AAAs (5). Furthermore, increased $18 \mathrm{~F}$-fluorodeoxyglucose (18F-FDG) uptake that is detected by positron emission tomography (PET) in AAAs has been demonstrated to be associated with inflammation, aortic wall instability and an increased AAA rupture risk $(6,7)$. The $\mathrm{MA}^{3} \mathrm{RS}$ study therefore extends the results of previous studies and shows that USPIO-enhanced MRI predicts the rate of AAA expansion, as well as the risk of AAA rupture and AAA repair. This novel finding is particularly important, especially in high-risk and unfit patients, as it can identify those individuals more likely to benefit from a prophylactic AAA repair, or those who can safely be managed conservatively. Future studies should verify the results of the $\mathrm{MA}^{3} \mathrm{RS}$ study and help establish this novel method of AAA rupture risk stratification.

Cite this article as: Paraskevas KI. Aortic wall inflammation: a more accurate predictor of aneurysm expansion and aneurysm rupture risk than aortic diameter? J Thorac Dis 2018;10(Suppl 33):S3865-S3866. doi: 10.21037/jtd.2018.09.36

\section{Acknowledgements}

None.

\section{Footnote}

Conflicts of Interest: The author has no conflicts of interest to declare.

\section{References}

1. Chaikof EL, Dalman RL, Eskandari MK, et al. The Society for Vascular Surgery practice guidelines on the care of patients with an abdominal aortic aneurysm. J Vasc Surg 2018;67:2-77.e2.

2. Ahmad M, Mistry R, Hodson J, et al. How Quickly Do Asymptomatic Infrarenal Abdominal Aortic Aneurysms Grow and What Factors Affect Aneurysm Growth Rates? Analysis of a Single Centre Surveillance Cohort Database. Eur J Vasc Endovasc Surg 2017;54:597-603.

3. Whittaker JD, Meecham L, Summerour V, et al. Outcome after Turndown for Elective Abdominal Aortic Aneurysm Surgery. Eur J Vasc Endovasc Surg 2017;54:579-86.

4. MA3RS Study Investigators. Aortic Wall Inflammation Predicts Abdominal Aortic Aneurysm Expansion, Rupture, and Need for Surgical Repair. Circulation 2017;136:787-97.

5. Paraskevas KI, Andrikopoulou M, Anastasakis E, et al. Oxidative stress in the pathogenesis of abdominal aortic aneurysms: a possible pathway for the effect of statins? Angiology 2010;61:226-7; author reply 227-8.

6. Reeps C, Essler M, Pelisek J, et al. Increased $18 \mathrm{~F}$-fluorodeoxyglucose uptake in abdominal aortic aneurysms in positron emission/computed tomography is associated with inflammation, aortic wall instability, and acute symptoms. J Vasc Surg 2008;48:417-23; discussion 424

7. Paraskevas KI, Mikhailidis DP, Veith FJ.

18F-fluorodeoxyglucose uptake in abdominal aortic aneurysms: a useful biomarker of AAA rupture risk. Biomed Res Int 2014;2014:930738. 